\title{
EFFECTS OF BLACK HOLES ON INFLATON PERTURBATION
}

\author{
HING-TONG CHO \\ Department of Physics, Tamkang University, Tamsui 251, Taiwan \\ htcho@mail.tku.edu.tw \\ KIN-WANG NG \\ Institute of Physics, Academia Sinica, Taipei 11529, Taiwan \\ nkw@phys.sinica.edu.tw \\ I-CHIN WANG \\ Department of Physics, National Taiwan Normal University, Taipei 116, Taiwan \\ wic@phys.sinica.edu.tw
}

\begin{abstract}
We calculate quantum fluctuations of a free scalar field in the Schwarzschild-de Sitter space-time, adopting the planar coordinates that is pertinent to the presence of a black hole in an inflationary universe. In a perturbation approach, doing expansion in powers of a small black hole event horizon compared to the de Sitter cosmological horizon, we obtain the scalar power spectrum.
\end{abstract}

Keywords: Inflation; density perturbation; black holes.

\section{Introduction}

The cosmic microwave background that we observe today is almost homogenous and isotropic. The background temperature in our sky is about $2.7 \mathrm{~K}$ with a tiny fluctuation at a level of about $10^{-5} \mathrm{~K}$. This is consistent with measurements of the matter content of the Universe, altogether prevailing a spatially flat universe. Inflation scenario can explain the homogeneity, the isotropy, and the flatness of the present Universe $^{1}$. Moreover, quantum fluctuations of the inflaton field during inflation can give rise to primordial density fluctuations with a nearly scale-invariant power spectrum which is consistent with the recent WMAP data on cosmic microwave background anisotropies $^{2}$. Therefore, a general assumption usually made in most cosmological models is that the background metric is homogenous and isotropic. For example, a de Sitter metric is used in the inflationary era and a flat Friedmann-RobertsonWalker metric is used in the subsequent hot big bang.

Apparently our present Universe is not so homogeneous and isotropic because we observe local non-linear structures such as stars, galaxies, clusters of galaxies, and very massive black holes. An appropriate space-time, for example, for a massive black hole sitting in the accelerating Universe, would be described by the 
Schwarzschild metric in the vicinity of the black hole and by the de Sitter metric at places far from the black hole. Presently, these local structures are decoupled from the Hubble flow, so it suffices to use the Friedmann-Robertson-Walker metric to study the large scale structures of the Universe. However, in the early Universe the gravitational effect of a black hole to the background metric may be important and should be addressed. For example, the existence of a black hole or a distribution of black holes at the onset of inflation can invalidate the use of a homogenous and isotropic background metric for the calculation of de Sitter quantum fluctuations. This also applies to the situation when we are very near to one of these black holes that still exists today or not.

The cosmic no hair conjecture infers that the inflationary universe approaches asymptotically the de Sitter space-time till the end of inflation ${ }^{3}$. Nevertheless, the effects of matter and space-time inhomogeneities to inflation should be considered. Several authors have studied the onset of inflation under inhomogeneous initial conditions to determine whether large inhomogeneity during the very early Universe can prevent the Universe from entering an inflationary era ${ }^{4}$. It was found that in some cases a large initial inhomogeneity may suppress the onset of inflation ${ }^{5}$. If the inflaton field is sufficiently inhomogeneous, the wormhole can form from collapsing vacuum energy density peaks before the inhomogeneity is damped by the exponential expansion ${ }^{6}$. In the case of inhomogeneities in a dust era before inflation, some inhomogeneities can collapse into a black-hole space-time ${ }^{7}$. Furthermore, for the inhomogeneities of the space-time itself, energies in the form of gravitational waves can also form a black-hole space-time ${ }^{8}$. As a consequence, at the onset of inflation, the distortion of the metric by these inhomogeneities should be taken into account. With these considerations in mind, we will investigate the quantum fluctuations of a free massless scalar field in the Schwarzschild-de Sitter (SdS) space-time.

\section{Black Hole Effects to Inflation}

We will investigate the quantum fluctuations of a free massless scalar field in the Schwarzschild-de Sitter (SdS) space-time ${ }^{9}$. We will use the planar coordinates for the SdS metric, which is given by

$$
d s^{2}=-f(r, \tau) d \tau^{2}+h(r, \tau)\left(d r^{2}+r^{2} d \Omega^{2}\right),
$$

where $d \tau=a^{-1}(\tau) d t$ is the conformal time and $a(t)=e^{H t}$. The $f$ and $h$ functions are given by

$$
f(r, \tau)=a^{2}(\tau)\left[1-\frac{G M}{2 a(\tau) r}\right]^{2}\left[1+\frac{G M}{2 a(\tau) r}\right]^{-2}, \quad h(r, \tau)=a^{2}(\tau)\left[1+\frac{G M}{2 a(\tau) r}\right]^{4}
$$

with the cosmic scale factor $a(\tau)=-1 /(H \tau)$, where $G=M_{\mathrm{Pl}}^{-2}, M$ is the mass of the black hole, and $H$ is the Hubble parameter for inflation. Here we use the convention with $c=\hbar=1$. 
Consider a massless scalar field $\phi$ which satisfies the Klein-Gordon equation in the SdS space-time,

$$
\partial_{\mu}\left(\sqrt{-g} g^{\mu \nu} \partial_{\nu} \phi\right)=0
$$

A unique mode function $\varphi_{k l m}(x)$ can be obtained once an appropriate vacuum is chosen. By using this mode function the scalar field can be quantized in the standard manner,

$$
\hat{\phi}(x)=\int_{0}^{\infty} d k \sum_{l m}\left[\hat{a}_{k \operatorname{lm}} \varphi_{k l m}(x)+\hat{a}_{k l m}^{\dagger} \varphi_{k l m}^{*}(x)\right]
$$

with the commutation relations:

$$
\begin{aligned}
& {\left[\hat{a}_{k l m}, \hat{a}_{k^{\prime} l^{\prime} m^{\prime}}\right]=\left[\hat{a}_{k l m}^{\dagger}, \hat{a}_{k^{\prime} l^{\prime} m^{\prime}}^{\dagger}\right]=0} \\
& {\left[\hat{a}_{k l m}, \hat{a}_{k^{\prime} l^{\prime} m^{\prime}}^{\dagger}\right]=\delta\left(k-k^{\prime}\right) \delta_{l l^{\prime}} \delta_{m m^{\prime}}}
\end{aligned}
$$

Now $\varphi_{k l m}(x)$ is the mode function, so it should also satisfy the Klein-Gordon equation. Since the space is spherically symmetric, one can write

$$
\varphi_{k l m}(x)=\varphi_{k l}(r, \tau) Y_{l m}(\theta, \phi) .
$$

The Klein-Gordon equation for the mode function is given by

$$
-\frac{1}{\sqrt{f h}} \partial_{\tau}\left(\sqrt{\frac{h^{3}}{f}} \partial_{\tau} \varphi_{k l}(r, \tau)\right)+\frac{1}{r^{2} \sqrt{f h}} \partial_{r}\left(r^{2} \sqrt{f h} \partial_{r} \varphi_{k l}(r, \tau)\right)-\frac{l(l+1)}{r^{2}} \varphi_{k l}(r, \tau)=0 .
$$

In terms of $\varphi_{k l}(r, \tau)$, the spectral function of the fluctuations of the quantum field $\phi(x)$ can be defined as

$$
S_{k l}(r, \tau) \equiv(2 l+1) j_{l}^{2}(k r) P_{k l}(r, \tau), \quad P_{k l}(r, \tau)=\frac{k}{4 \pi j_{l}^{2}(k r)}\left|\varphi_{k l}(r, \tau)\right|^{2}
$$

Perturbatively,

$$
\begin{aligned}
P_{k l}(r, \tau) & =\frac{k}{4 \pi j_{l}^{2}(k r)}\left|\varphi_{k l}^{(0)}(r, \tau)+\varphi_{k l}^{(1)}(r, \tau)+\cdots\right|^{2} \\
& =P_{k l}^{(0)}(r, \tau)\left[1+\epsilon \Delta_{k l}^{(1)}(r, \tau)+\cdots\right]
\end{aligned}
$$

where $\epsilon=G M H \ll 1$ and we have defined

$$
\begin{aligned}
& P_{k l}^{(0)}(r, \tau)=\frac{k}{4 \pi j_{l}^{2}(k r)}\left|\varphi_{k l}^{(0)}(r, \tau)\right|^{2} \\
& \Delta_{k l}^{(1)}(r, \tau)=2 \epsilon^{-1}\left|\varphi_{k l}^{(0)}(r, \tau)\right|^{-2} \operatorname{Re}\left[\varphi_{k l}^{(0)}(r, \tau) \varphi_{k l}^{(1)^{*}}(r, \tau)\right] .
\end{aligned}
$$




\subsection{Zeroth order}

To the zeroth order we have

$$
\partial_{\tau}^{2} \varphi_{k l}^{(0)}(r, \tau)-\frac{2}{\tau} \partial_{\tau} \varphi_{k l}^{(0)}(r, \tau)-\partial_{r}^{2} \varphi_{k l}^{(0)}(r, \tau)-\frac{2}{r} \partial_{r} \varphi_{k l}^{(0)}(r, \tau)+\frac{l(l+1)}{r^{2}} \varphi_{k l}^{(0)}(r, \tau)=0
$$

and the general solution is found to be

$$
\varphi_{k l}^{(0)}(r, \tau)=k^{2} j_{l}(k r) \varphi_{k l}^{(0)}(\tau)
$$

where the choice of the Bunch-Davies vacuum selects the mode function:

$$
\varphi_{k l}^{(0)}(\tau)=-\frac{H \tau}{k \sqrt{\pi k}}\left(1-\frac{i}{k \tau}\right) e^{-i k \tau}
$$

and hence the zeroth order power spectrum is

$$
P_{k l}^{(0)}(r, \tau)=\frac{k}{4 \pi j_{l}^{2}(k r)}\left|\varphi_{k l}^{(0)}(r, \tau)\right|^{2}=\frac{H^{2}}{4 \pi^{2}}\left(1+k^{2} \tau^{2}\right) .
$$

\subsection{First order}

To the next order, we have

$$
\partial_{\tau}^{2} \varphi_{k l}^{(1)}(r, \tau)-\frac{2}{\tau} \partial_{\tau} \varphi_{k l}^{(1)}(r, \tau)-\partial_{r}^{2} \varphi_{k l}^{(1)}(r, \tau)-\frac{2}{r} \partial_{r} \varphi_{k l}^{(1)}(r, \tau)+\frac{l(l+1)}{r^{2}} \varphi_{k l}^{(1)}(r, \tau)=J_{1},
$$

where

$$
\begin{aligned}
J_{1}(r, \tau) & =\frac{4 \epsilon \tau}{r}\left[\partial_{\tau}^{2} \varphi_{k l}^{(0)}(r, \tau)-\frac{1}{\tau} \partial_{\tau} \varphi_{k l}^{(0)}(r, \tau)\right] \\
& =\frac{4 \epsilon H \tau^{2}}{\sqrt{\pi} r}\left[k^{2} j_{l}(k r)\left(k^{\frac{1}{2}} e^{-i k \tau}\right)\right] .
\end{aligned}
$$

To solve this inhomogeneous equation, we use the Green's function $G\left(r, \tau ; r^{\prime}, \tau^{\prime}\right)$ which satisfies the equation,

$$
\partial_{\tau}^{2} G-\frac{2}{\tau} \partial_{\tau} G-\partial_{r}^{2} G-\frac{2}{r} \partial_{r} G+\frac{l(l+1)}{r^{2}} G=\frac{\delta\left(r-r^{\prime}\right) \delta\left(\tau-\tau^{\prime}\right)}{r^{2}} .
$$

Using the completeness property of the spherical Bessel functions,

$$
\int_{0}^{\infty} d k k^{2}\left[\sqrt{\frac{2}{\pi}} r j_{l}(k r)\right]\left[\sqrt{\frac{2}{\pi}} r^{\prime} j_{l}\left(k r^{\prime}\right)\right]=\delta\left(r-r^{\prime}\right),
$$

and taking

$$
G_{l}\left(r, \tau ; r^{\prime}, \tau^{\prime}\right)=\int_{0}^{\infty} d k k^{2} g_{k}\left(\tau, \tau^{\prime}\right) j_{l}(k r) j_{l}\left(k r^{\prime}\right),
$$

Eq. (18) becomes

$$
\partial_{\tau}^{2} g_{k}-\frac{2}{\tau} \partial_{\tau} g_{k}+k^{2} g_{k}=\frac{2}{\pi} \delta\left(\tau-\tau^{\prime}\right)
$$


For the retarded Green's function, $g_{k}=0$ for $\tau^{\prime}>\tau>\tau_{i}$, where $\tau_{i}$ denotes an initial time when the source begins to operate. For $0>\tau>\tau^{\prime}$,

$$
\begin{aligned}
g_{k}\left(\tau, \tau^{\prime}\right)=\frac{i}{2 \tau^{\prime 2} k^{3}}[ & (-k \tau)^{\frac{3}{2}} H_{\frac{3}{2}}^{(1)}(-k \tau)\left(-k \tau^{\prime}\right)^{\frac{3}{2}} H_{\frac{3}{2}}^{(2)}\left(-k \tau^{\prime}\right) \\
& \left.-\left(-k \tau^{\prime}\right)^{\frac{3}{2}} H_{\frac{3}{2}}^{(1)}\left(-k \tau^{\prime}\right)(-k \tau)^{\frac{3}{2}} H_{\frac{3}{2}}^{(2)}(-k \tau)\right] .
\end{aligned}
$$

Then,

$$
\begin{aligned}
& \varphi_{k l}^{(1)}(r, \tau) \\
= & \int_{0}^{\infty} d r^{\prime} r^{\prime 2} \int_{\tau_{i}}^{0} d \tau^{\prime} G\left(r, \tau ; r^{\prime}, \tau^{\prime}\right) J_{1}\left(r^{\prime}, \tau^{\prime}\right) \\
= & \frac{2 i \epsilon H k^{\frac{5}{2}}}{\sqrt{\pi}} \int_{0}^{\infty} \frac{d k^{\prime}}{k^{\prime}} j_{l}\left(k^{\prime} r\right) \int_{0}^{\infty} d r^{\prime} r^{\prime} j_{l}\left(k r^{\prime}\right) j_{l}\left(k^{\prime} r^{\prime}\right) \int_{\tau_{i}}^{\tau} d \tau^{\prime} e^{-i k \tau^{\prime}} \times \\
& {\left[\left(-k^{\prime} \tau\right)^{\frac{3}{2}} H_{\frac{3}{2}}^{(1)}\left(-k^{\prime} \tau\right)\left(-k^{\prime} \tau^{\prime}\right)^{\frac{3}{2}} H_{\frac{3}{2}}^{(2)}\left(-k^{\prime} \tau^{\prime}\right)\right.} \\
& \left.-\left(-k^{\prime} \tau^{\prime}\right)^{\frac{3}{2}} H_{\frac{3}{2}}^{(1)}\left(-k^{\prime} \tau^{\prime}\right)\left(-k^{\prime} \tau\right)^{\frac{3}{2}} H_{\frac{3}{2}}^{(2)}\left(-k^{\prime} \tau\right)\right] .
\end{aligned}
$$

This is in the form of

$$
\varphi_{k l}^{(1)}(r, \tau)=\epsilon \int_{0}^{\infty} \frac{d k^{\prime}}{k^{\prime}}\left[\alpha_{k k^{\prime} l}(\tau) \varphi_{k^{\prime} l}^{(0)}(r, \tau)+\beta_{k k^{\prime} l}(\tau) \varphi_{k^{\prime} l}^{(0)^{*}}(r, \tau)\right],
$$

where

$$
\begin{aligned}
& \alpha_{k k^{\prime} l}(\tau)=-\left(\frac{4 i}{\pi}\right) k^{\frac{5}{2}} k^{\prime \frac{1}{2}} \int_{0}^{\infty} d r^{\prime} r^{\prime} j_{l}\left(k r^{\prime}\right) j_{l}\left(k^{\prime} r^{\prime}\right) \int_{\tau_{i}}^{\tau} d \tau^{\prime}\left(k^{\prime} \tau^{\prime}+i\right) e^{-i\left(k-k^{\prime}\right) \tau^{\prime}} \\
& \beta_{k k^{\prime} l}(\tau)=\left(\frac{4 i}{\pi}\right) k^{\frac{5}{2}} k^{\prime \frac{1}{2}} \int_{0}^{\infty} d r^{\prime} r^{\prime} j_{l}\left(k r^{\prime}\right) j_{l}\left(k^{\prime} r^{\prime}\right) \int_{\tau_{i}}^{\tau} d \tau^{\prime}\left(k^{\prime} \tau^{\prime}-i\right) e^{-i\left(k+k^{\prime}\right) \tau^{\prime}}
\end{aligned}
$$

Now we calculate the leading correction (11) to the power spectrum. Substituting Eq. (13) in Eq. (24), we have

$$
\begin{aligned}
\Delta_{k l}^{(1)}(r, \tau)= & \int_{0}^{\infty} \frac{d k^{\prime}}{k^{\prime}} \frac{k^{\prime 2}}{k^{2}} \frac{j_{l}\left(k^{\prime} r\right)}{j_{l}(k r)}\left|\varphi_{k l}^{(0)}(\tau)\right|^{-2} \times \\
& 2 \operatorname{Re}\left[\alpha_{k k^{\prime} l}^{*}(\tau) \varphi_{k l}^{(0)}(\tau) \varphi_{k^{\prime} l}^{(0) *}(\tau)+\beta_{k k^{\prime} l}^{*}(\tau) \varphi_{k l}^{(0)}(\tau) \varphi_{k^{\prime} l}^{(0)}(\tau)\right] .
\end{aligned}
$$

As $\tau \rightarrow 0$, this becomes

$$
\Delta_{k l}^{(1)}(r, 0)=\int_{0}^{\infty} \frac{d k^{\prime}}{k^{\prime}}\left(\frac{k}{k^{\prime}}\right)^{\frac{1}{2}} \frac{j_{l}\left(k^{\prime} r\right)}{j_{l}(k r)} 2 \operatorname{Re}\left[\alpha_{k k^{\prime} l}(0)-\beta_{k k^{\prime} l}(0)\right] .
$$


Here we obtain from Eqs. (25) and (26) that

$$
\begin{aligned}
& 2 \operatorname{Re}\left[\alpha_{k k^{\prime} l}(0)-\beta_{k k^{\prime} l}(0)\right] \\
= & \frac{16}{\pi}\left(\frac{k}{k^{\prime}}\right)^{\frac{1}{2}} k^{2} \int_{0}^{\infty} d r^{\prime} r^{\prime} j_{l}\left(k r^{\prime}\right) j_{l}\left(k^{\prime} r^{\prime}\right) \times \\
& \left(\frac{k^{2}}{k^{\prime 2}}-1\right)^{-2}\left\{\cos \left(k^{\prime} \tau_{i}\right)\left[2 \sin \left(k \tau_{i}\right)-\left(\frac{k^{2}}{k^{\prime 2}}-1\right) k \tau_{i} \cos \left(k \tau_{i}\right)\right]\right. \\
& \left.+\sin \left(k^{\prime} \tau_{i}\right)\left[\frac{k}{k^{\prime}}\left(\frac{k^{2}}{k^{\prime 2}}-3\right) \cos \left(k \tau_{i}\right)-\left(\frac{k^{2}}{k^{\prime 2}}-1\right) k^{\prime} \tau_{i} \sin \left(k \tau_{i}\right)\right]\right\} .
\end{aligned}
$$

This quantity diverges as $k \rightarrow k^{\prime}$. However, we find that the integral in Eq. (28) is finite and weakly dependent on $r$. In the limit of $r \rightarrow 0$, it can be approximated by

$$
\Delta_{k l}^{(1)}(r, 0) \simeq \int_{0}^{\infty} \frac{d k^{\prime}}{k^{\prime}}\left(\frac{k^{\prime}}{k}\right)^{l-\frac{1}{2}} 2 \operatorname{Re}\left[\alpha_{k k^{\prime} l}(0)-\beta_{k k^{\prime} l}(0)\right] .
$$

In Fig. 1, we plot the asymptotic values $\Delta_{k l}^{(1)}(r, 0)$ for $H r=0.01,0.1$ against $l$ and $k / H$ for $1<l<50$ and $1<k / H<50$. Note that if inflation lasts for about 60 e-folds, $r=H^{-1}$ will be about the size of the present Universe and $H r<1$ corresponds to sub-horizon length scales.

\section{Conclusions}

We have presented a perturbation method to compute the effect of the presence of a black hole in the de Sitter space to the quantum fluctuations of a free massless scalar field. The method is valid as long as the expansion parameter $\epsilon \equiv G M H \ll 1$, i.e., the size of the black hole event horizon is smaller than that of the de Sitter cosmological horizon. The calculation can be easily applied to a vector field or a gravitational wave. Here the first-order contribution is computed and the results are given in the assumption that the black hole is located at the origin of the coordinates. Higher-order corrections can be worked out perturbatively though complicated. It would be interesting to consider the effect due to a distribution of black holes in the de Sitter space. In fact, the perturbation can be in a form of cosmological defects such as monopoles, cosmic strings, or domain walls.

Let us briefly discuss some cosmological implications of the results that we have obtained in this work. Assume that inflation lasts for about 60 e-folds. Then, the wavelength of the Fourier mode with $k / H=1$ is about the size of the present Universe. If the location of the black hole that exists during inflation is near the Earth, the suppressed power of the first-order correction to the de Sitter inflaton fluctuations in low $l$ and low $k$ regions may result in a blue-tilted density power spectrum on large angular scales. This in turn gives rise to a suppression of the largescale cosmic microwave background anisotropy that may be relevant to the observed low quadrupole in the WMAP cosmic microwave background anisotropy data?. A detailed calculation of the effect to the cosmic microwave background anisotropy 

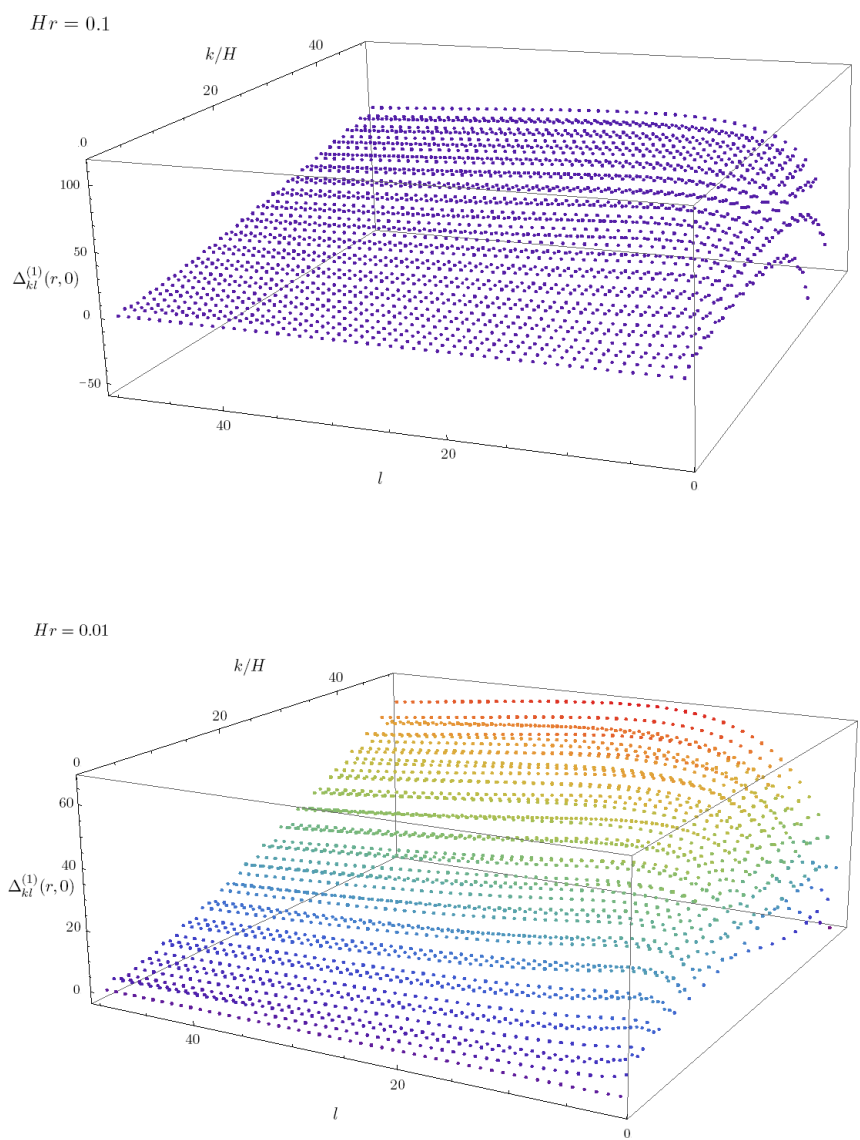

Fig. 1. Three-dimensional plots of the asymptotic values $\Delta_{k l}^{(1)}(r, 0)$ for $H r=0.01$ (lower) and $H r=0.1$ (upper) against $l$ and $k / H$ for $1<l<50$ and $1<k / H<50$.

would be interesting, including the case that the black hole locates somewhere else in the Universe. It is worth noting that the present work gives a realization of the general discussions in Ref. ${ }^{10}$ about the potentially observable effects of a small violation of translational invariance during inflation, as characterized by the presence of a preferred point, line, or plane. This violation may induce derivations from pure statistical isotropy of cosmological perturbations, thus leaving anomalous imprints on the cosmic microwave background anisotropy ${ }^{10}$.

\section{Acknowledgments}

This work was supported in part by the National Science Council, Taiwan, ROC under Grant Nos. NSC 96-2112-M-032-006-MY3 (HTC), NSC 95-2112-M-001-052MY3 (KWN), NSC 97-2112-M-003-004-MY3 (ICW), and the National Center for Theoretical Sciences, Taiwan, ROC. 


\section{References}

1. For reviews see: K. A. Olive, Phys. Rep. 190, 307 (1990); D. H. Lyth and A. Riotto, Phys. Rep. 314, 1 (1999).

2. E. Komatsu et al., Astrophys. J. Suppl. 180, 330 (2009).

3. G. W. Gibbons and S. W. Hawking, Phys. Rev. D 15, 2738 (1977).

4. H. Kurki-Suonio, J. Centrella, R. A. Matzner, and J. R. Wilson, Phys. Rev. D 35, 435 (1987); D. S. Goldwirth and T. Piran, Phys. Rev. D 40, 3263 (1989); P. Laguna, H. Kurki-Suonio, and R. A. Matzner, Phys. Rev. D 44, 3077 (1991).

5. D. S. Goldwirth and T. Piran, Phys. Rev. Lett. 64, 2852 (1990); E. Calzetta and M. Sakellariadou, Phys. Rev. D 45, 2802 (1992).

6. K. A. Holcomb, S. J. Park, and E. T. Vishniac, Phys. Rev. D 39, 1058 (1989).

7. D. Garfinkle and C. Vuille, Gen. Relativ. Gravit. 23, 471 (1991); K. Nakao, Gen. Relativ. Gravit. 24, 1069 (1992).

8. K. Nakao, K. Maeda, T. Nakamura, and K. Oohara, Phys. Rev. D 47, 3194 (1993).

9. H.-T. Cho, K.-W. Ng, and I-C. Wang, arXiv:0905.2041.

10. S. M. Carroll, C.-Y. Tseng, and M. B. Wise, Phys. Rev. D 81, 083501 (2010). 